Portland State University

PDXScholar

$5-13-2013$

Photonic Near-Field Imaging in Multiphoton

Photoemission Electron Microscopy

Joseph Fitzgerald

Portland State University

Robert Campbell Word

Portland State University

S. D. Saliba

Portland State University

Rolf Könenkamp

Portland State University, rkoe@pdx.edu

Follow this and additional works at: https://pdxscholar.library.pdx.edu/phy_fac

Part of the Physics Commons

Let us know how access to this document benefits you.

Citation Details

Fitzgerald, J. P. S., et al. "Photonic near-field imaging in multiphoton photoemission electron microscopy." Physical Review B 87.20 (2013): 205419.

This Article is brought to you for free and open access. It has been accepted for inclusion in Physics Faculty Publications and Presentations by an authorized administrator of PDXScholar. Please contact us if we can make this document more accessible: pdxscholar@pdx.edu. 


\title{
gु \\ Photonic near-field imaging in multiphoton photoemission electron microscopy
}

\author{
J. P. S. Fitzgerald, ${ }^{*}$ R. C. Word, S. D. Saliba, and R. Könenkamp \\ Department of Physics, Portland State University, 1719 SW 10th Avenue, Portland, OR 97201, USA
}

(Received 15 February 2013; published 13 May 2013)

\begin{abstract}
We report the observation of optical near fields in a photonic waveguide of conductive indium tin oxide (ITO) using multiphoton photoemission electron microscopy (PEEM). Nonlinear two-photon photoelectron emission is enhanced at field maxima created by interference between incident 410-nm and coherently excited guided photonic waves, providing strong phase contrast. Guided modes are observed under both transverse magnetic field (TM) and transverse electric field (TE) polarized illuminations and are consistent with classical electromagnetic theory. Implications on the role of multiphoton PEEM in optical near-field imaging are discussed.
\end{abstract}

DOI: 10.1103/PhysRevB.87.205419

PACS number(s): 68.37.Xy, 42.25.-p, 42.82.Et, 78.67.-n

\section{INTRODUCTION}

Multiphoton photoemission relies on the nonlinear interaction of high intensity light at a solid surface to induce electron photoemission even if photon energies are below the work-function threshold of the material. ${ }^{1}$ Imaging these photoelectrons in a photoemission electron microscope (PEEM) reveals a spatial map of the emission rate distribution. Emission locations can be linked to work-function differences, surface features, local electric fields, and electron densities. ${ }^{2,3}$ On metal surfaces emission sites can also be associated with surface plasmon polaritons (SPPs). ${ }^{4-6}$ SPPs result from the coupling of photons and conduction electrons in a metal and are confined to the surface near region.

Photonic structures based on dielectric waveguides similarly confine electromagnetic fields but without the resonant coupling to the free electron gas, as the resonance frequency of the electrons in a dielectric is typically far below the used photon frequencies. So far, imaging of photonic near fields with PEEM is not reported because the typical transparent dielectric does not have the right combination of high transparency and electron density. A likely candidate is a transparent conducting oxide such as indium-tin oxide (ITO). ${ }^{7}$

In this paper we report PEEM carried out with 100-fs pulses of 410-nm light. We used ITO, which has a work function of approximately $4.2 \mathrm{eV}^{8}$ Figure 1 shows the dispersion relationship of this material. At $410 \mathrm{~nm}$, ITO exhibits radiative photonic waves, above the light line in the dispersion relation. This response is clearly distinct from a plasmonic response, which can be expected for wavelengths greater than 1.5 microns ${ }^{11}$-i.e., at wavelengths greater than the surface plasmon resonance wavelength. At those wavelengths the dispersion curve lies below the light line.

In the following, we present multiphoton PEEM images of a simple photonic structure with nanometer dimensions. We then develop a classical model to analyze waves propagating parallel to the surface. We use it to show that the properties of the observed waves are consistent with dielectric waveguide modes. This finding has implications for the interpretation of multiphoton PEEM and the usefulness of the microscopy technique for more general, high-resolution imaging in nanophotonics, which we discuss in the last section.

\section{EXPERIMENTAL DETAILS}

\section{A. Photonic nanostructure}

We used 0.2-mm-thick borosilicate glass wafers covered with a thin layer of ITO with sheet resistivity of $15-20 \Omega / \square$ and thickness $t_{\mathrm{ITO}}=290 \pm 20 \mathrm{~nm}$, obtained from SPI Supplies. At a wavelength of $410 \mathrm{~nm}$, the real parts of the refractive indices are $n_{\text {ITO }}=2.14 \pm 0.01$ and $n_{\text {glass }}=1.53 \pm 0.01$. The imaginary part of the refractive index of ITO is $\kappa \approx 0.025,{ }^{12,13}$ corresponding to an absorption coefficient of $\alpha \approx 8000 \mathrm{~cm}^{-1}$. The optical properties of ITO are known to vary with film thickness. $^{14}$

Using a FEI Strata 237 focused ion beam (FIB), we milled a $6.0 \times 0.4-\mu \mathrm{m}^{2}$ slit of depth $\approx 320 \mathrm{~nm}$ into the ITO. In the PEEM, this slit was oriented perpendicularly to the laser beam direction and served to couple incident light into the ITO layer, as shown in Fig. 2. A wide, shallow trench measuring $2.0 \times 5.0 \mu \mathrm{m}^{2}$ perpendicular to the slit was milled to produce a region of ITO with a reduced thickness of approximately $240 \mathrm{~nm}$.

\section{B. Multiphoton PEEM apparatus}

Incident light is generated by a Spectra-Physics Mai Tai Ti:sapphire laser, producing $11-\mathrm{nJ}, 80$-fs pulses at $820 \mathrm{~nm}$ at a frequency of $80 \mathrm{MHz}$. These pulses are up-converted to 2-nJ, 410-nm pulses using a Del Mar Photonics second harmonic generator. The optical bandwidth $\Delta v / v$ of the pulse is less than $3 \%$. Thus, nearly monochromatic conditions are obtained. The laser pulses can be adjusted to be either vertically (TE) or horizontally (TM) polarized using a tunable wave plate.

The polarized beam is focused to a $100-\mu$ m-diameter spot, encountering the sample at an angle of $60^{\circ}$ to the sample surface normal, as shown in Fig. 2. The photon flux at the sample surface is approximately 0.005 photon $\mathrm{nm}^{-2} \mathrm{fs}^{-1}$ at peak intensity. Photoelectrons, at a yield of less than 1 per pulse, are collected and imaged by an aberration-correction PEEM in which spherical and chromatic aberration are corrected using a hyperbolic electron mirror. Enhanced brightness and contrast gained by aberration correction can significantly reduce imaging times over uncorrected PEEM. Details of the microscope are described in Refs. 15 and 16. 


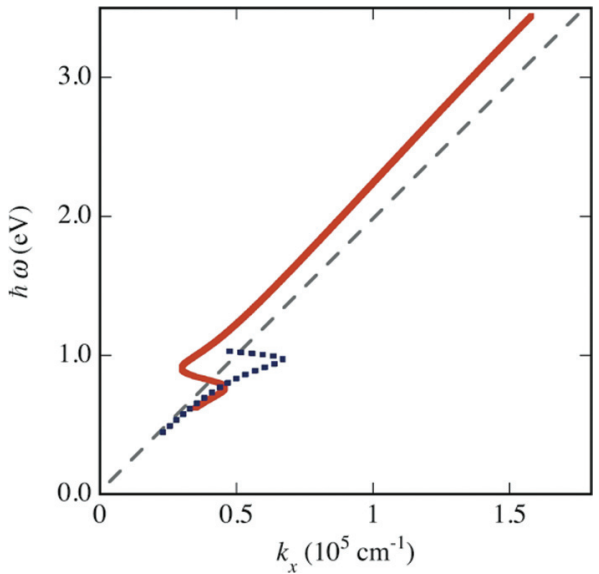

FIG. 1. (Color online) Dispersion relationship for SPPs propagating at the interface of indium-tin oxide with vacuum. Waves observed in PEEM are excited with $410-\mathrm{nm}$ light at $3 \mathrm{eV}$, well above the surface plasma frequency in the infrared $(0.8-1.0 \mathrm{eV})$. Calculated from optical constants of Refs. 9 (red line) and 10 (blue dotted line).

\section{RESULTS AND DISCUSSION}

Figure 3(a) shows a PEEM micrograph of the ITO structure obtained with $\mathrm{CW}$ illumination at $244 \mathrm{~nm}$ from a frequency doubled Ar-ion laser. The photon energy for this ultraviolet light is $5.1 \mathrm{eV}$, which is larger than the work-function energy of ITO, and the PEEM image is therefore obtained in a singlephoton photoemission process. Mostly topological contrast is obtained in this imaging mode. The center region $\mathrm{B}$ is particularly bright due to surface roughness created by FIB milling.

When pulsed light at a wavelength of $410 \mathrm{~nm}$ is used, the character of the PEEM image changes significantly, as in Figs. 3(b) and 3(c). A wavelength of $410 \mathrm{~nm}$ corresponds to a photon energy of $3.02 \mathrm{eV}$. As this is less than the work function, nonlinear photoemission must be assumed. In these images some of the topological features are still recognizable, but many edges now appear highlighted and wavelike patterns with wave fronts perpendicular to the illumination direction

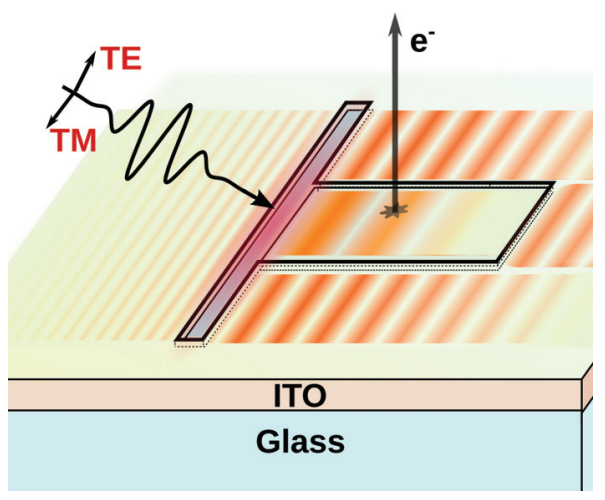

FIG. 2. (Color online) Photonic structure imaged by PEEM. A $6.0 \times 0.4-\mu \mathrm{m}^{2}$ slit and a shallow, $2.0 \times 5.0-\mu \mathrm{m}^{2}$ trench milled into ITO thin film on glass. Structure is illuminated by light at $60^{\circ}$ to surface normal, and emitted electrons are imaged using a photoemission electron microscope (PEEM).

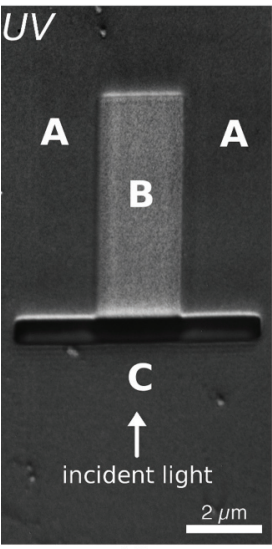

(a)

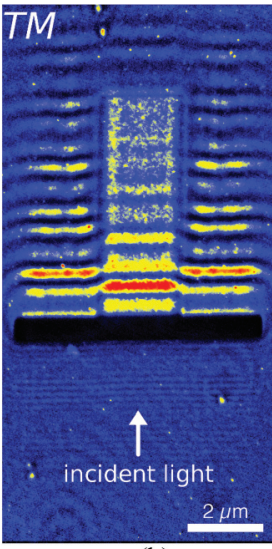

(b)

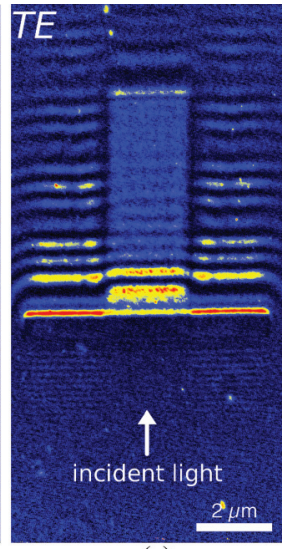

(c)
FIG. 3. (Color online) PEEM images. Laser is obliquely incident from the bottom edge at $60^{\circ}$ to surface normal. Regions of interest are indicated by A, B, and C. (a) Single-photon, 244-nm, continuouswave (cw), TM polarized illumination. (b),(c) Two-photon, 410-nm, 100-fs pulsed, (b) TM and (c) TE polarized illumination; shown in false color (online).

are now visible. Edges facing the incident light have enhanced emission compared to edges at other orientations, especially in TE polarization. Strong localized emission due to surface inhomogeneities or deposited particles are evident across the sample surface, with varying intensities. These localized features exhibit the signatures of diffraction and near-field radiative modes.

Wavelike patterns surround the structure in Figs. 3(b) and 3(c). The patterns exhibit different spacings, decay lengths, and amplitude variations in different regions and for different polarizations. With closer inspection, all regions appear to have an offset maximum and beating phenomena. Three different wave fields can be identified; these have been labeled A, B, and $\mathrm{C}$ in Fig. 3. Region A is in the forward direction of the incident laser beam, i.e., above the slit and outside the trench area. Region $\mathrm{B}$ is the trench center region, and region $\mathrm{C}$ is in the reverse direction incident to the beam, i.e., below the slit. The average intensity profiles across each region and polarization are shown in Fig. 4.

These wave patterns are well explained as an interference between the guided modes in the ITO layer and the incident light. The interference, illustrated in Fig. 5, is similar to those found in plasmonic metal structures, as reported, for example, in Refs. 17-19. There are, however, significant differences between the plasmonic and the photonic cases: Due to the lower electron densities in the ITO material and the much lower optical absorptivities in ITO as compared to typical metals, the electron emission rates are lower in the photonic case. At the same time the propagation length in the photonic case is comparable to thick silver, the best of the optical plasmonic metals. ${ }^{20}$ Typically we can observe the wave patterns to extend over distances of some 10 micrometers.

\section{A. Interference model}

In the following, we develop a scalar wave model to analyze these interference patterns in regions $\mathrm{A}, \mathrm{B}$, and $\mathrm{C}$ of Figs. 3(b) and 3(c). The bright areas of the observed 

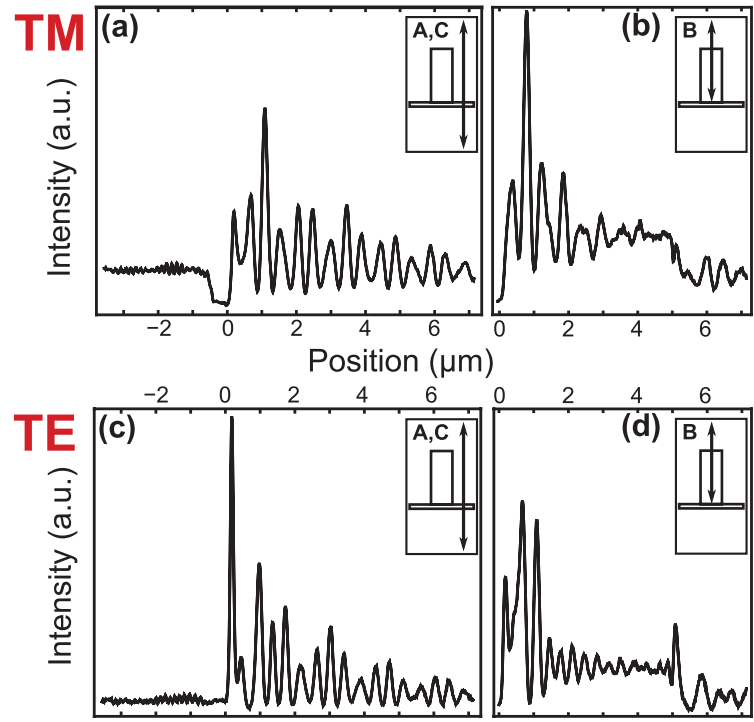

FIG. 4. (Color online) Two-photon PEEM interference pattern profiles, averaged from the regions marked in insets. (a),(b) TM polarized, (c),(d) TE polarized.

interference pattern occur where the incident and guided waves interfere constructively. We assume two guided waves, $E_{1}$ and $E_{2}$, corresponding to the minimum number of sources needed to explain the beating patterns in Fig. 4 (especially evident in the reverse direction). The incident and guided waves have propagation vectors $\mathbf{k}$ and $\mathbf{k}_{j}$ with in-plane components $k \sin \theta$ and $\pm k N_{j}$, respectively, where $k=2 \pi / 410 \mathrm{~nm}, \omega=c k$, $\theta=60^{\circ}$, and $\pm N_{j}$ is the effective refractive index of guided wave $j=1,2$ traveling in the forward $(+)$ or reverse $(-)$ direction. We first consider the interference pattern in the forward direction.

At surface location $y$ and time $t$ the incident wave, with field amplitude $E_{\text {inc }}(y, t)=A e^{i(k y \sin \theta-\omega t)}$, interferes with the $j$ th guided wave $E_{j}(y, t)=B_{j} e^{i\left( \pm k y N_{j}-\omega t\right)}$. The resulting electromagnetic field intensity distribution $I_{\mathrm{EM}}=\left\|E_{\mathrm{tot}}\right\|^{2}$, where $E_{\mathrm{tot}}=E_{\mathrm{inc}}+E_{1}+E_{2}$, has interference maxima separated by distances $d_{j}=2 \pi / \Delta k_{j}=2 \pi / k\left(N_{j} \mp \sin \theta\right)$ and $d_{3}=2 \pi / k\left(N_{2}-N_{1}\right)$, where $N_{2}>N_{1}$. The probability of two-photon photoemission is proportional to the square of the electromagnetic field intensity, or $I_{\mathrm{PE}}(t)=I_{\mathrm{EM}}^{2}=\left\|E_{\mathrm{tot}}\right\|^{4}$. The additional square introduces additional interference terms,

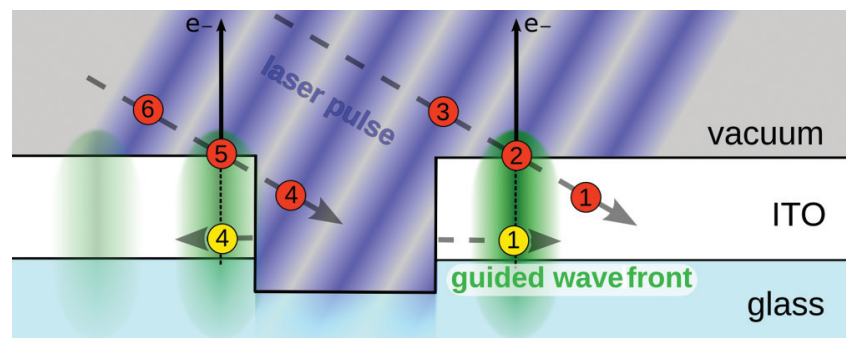

FIG. 5. (Color online) Image formation model for near-field enhancement in multiphoton PEEM. Incident wave fronts 1 and 4 excite guided wave fronts 1 and 4 . These propagate away from the slit and interfere constructively with the next incident wave fronts 2 and 5, enhancing the photoelectron emission rate there.
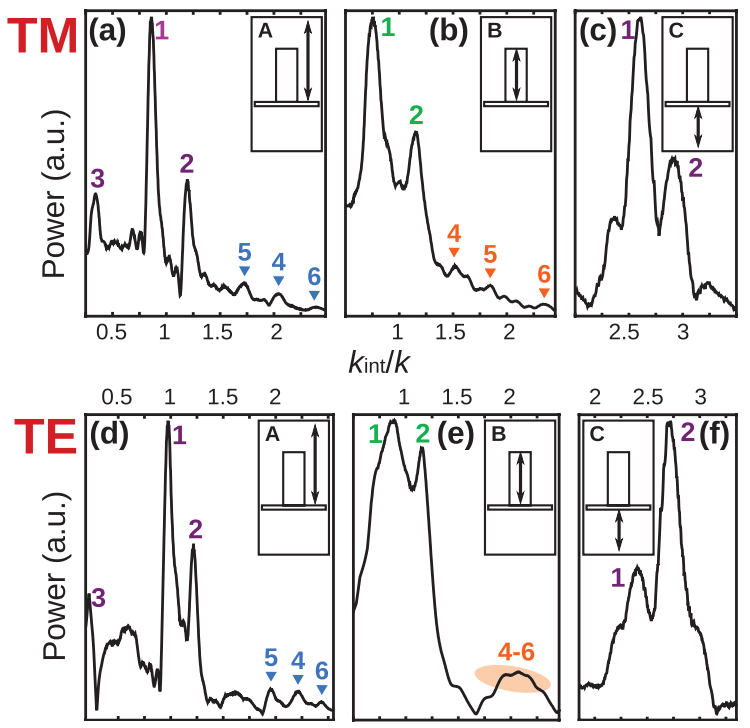

FIG. 6. (Color online) Fourier transform spectrum of Fig. 4, where $k=2 \pi / 410 \mathrm{~nm}$. Six interference peaks are highlighted in each spectrum, with signals 4-6 unique to multiphoton interference. The six spectra are (a)-(c) TM polarized, (d)-(f) TE polarized; from the regions marked in insets.

as we will see in the following. Integrating the photoemission intensity over time $t$ produces the photoelectron interference pattern,

$$
I_{\mathrm{PE}}=\left\|A e^{i k y \sin \theta}+B_{1} e^{i k y N_{1}}+B_{2} e^{i k y N_{2}}\right\|^{4} .
$$

A spatial Fourier transform of the intensity distribution offers more insight. The one-dimensional Fourier transform of the patterns in the three regions are shown in Fig. 6, and the results of the analysis are summarized in Table I. The results can be explained as follows: There are a total of at most six different wave numbers $\Delta k$ in the observed interference pattern. These wave numbers are apparent as peaks in the spatial Fourier transform.

As the in-coupling of the guided wave at the slit is not very efficient, the amplitude of the incident wave is in all cases larger than that of the guided waves $A>B_{j}$. The most intense signals, therefore, are $k_{\text {int, } j}=k\left(N_{j}-\sin \theta\right)$, $j=1,2$ with intensities proportional to $A^{3} B_{j}$. The next strongest signals, with intensities proportional to $A^{2} B_{i} B_{j}$, are $k_{\text {int }, 3}=k\left(N_{2}-N_{1}\right)$, and $k_{\text {int, } 4-6}=k\left(N_{i}+N_{j}-2 \sin \theta\right)$. Signals 4 through 6 are unique to multiphoton photoemission interference.

These six signals are labeled in the experimental fast Fourier transforms (FFTs), Fig. 6. Six additional signals predicted by the Fourier transform of Eq. (1) are below the noise threshold of the PEEM image. Additionally, the amplitudes $B_{j}$ may contain attenuation and offset factors that reduce intensity, especially in signals 3 and 6 . The results of the analysis carried out for regions $\mathrm{A}$ and $\mathrm{B}$ can be adapted to the reverse modes, apparent in region $\mathrm{C}$, with the substitution $\sin \theta \rightarrow-\sin \theta$.

Identifying all the peaks in the Fourier transforms in Fig. 6 allows an accurate computation of the effective indices. This calculation is highly redundant because a large number of parameters of the system is accessible from the Fourier transform. This gives us a means to check on the overall 
TABLE I. Theoretical interference model signals, where $k_{\text {int }}$ is the interference wave number, obtained from the Fourier transform of Eq. (1) and roughly sorted by intensity assuming $A>B_{1}>B_{2}$ and $N_{2}>N_{1}$. Signals 4-6 are unique to multiphoton photoemission.

\begin{tabular}{|c|c|c|c|c|c|c|}
\hline \multirow[b]{2}{*}{ Label } & \multicolumn{3}{|c|}{ Single-photon accessible } & \multicolumn{3}{|c|}{ Multiphoton only } \\
\hline & 1 & 2 & 3 & 4 & 5 & 6 \\
\hline Amplitude & $A^{3} B_{1}$ & $A^{3} B_{2}$ & $A^{2} B_{1} B_{2}$ & $A^{2} B_{1}^{2}$ & $A^{2} B_{2}^{2}$ & $A^{2} B_{1} B_{2}$ \\
\hline Forward $k_{\text {int }}$ & $k\left(N_{1}-\sin \theta\right)$ & $k\left(N_{2}-\sin \theta\right)$ & $k\left(N_{2}-N_{1}\right)$ & $2 k\left(N_{1}-\sin \theta\right)$ & $2 k\left(N_{2}-\sin \theta\right)$ & $k\left(N_{1}+N_{2}-2 \sin \theta\right)$ \\
\hline Reverse $k_{\text {int }}$ & $k\left(N_{1}+\sin \theta\right)$ & $k\left(N_{2}+\sin \theta\right)$ & $k\left(N_{2}-N_{1}\right)$ & $2 k\left(N_{1}+\sin \theta\right)$ & $2 k\left(N_{2}+\sin \theta\right)$ & $k\left(N_{1}+N_{2}+2 \sin \theta\right)$ \\
\hline
\end{tabular}

consistency of our model. With two $N_{j}$ for each surface region ( $\mathrm{A}$ and $\mathrm{B}$ ) and polarization (TM, TE), a total of eight modes in the forward direction is obtained. There are also four modes in the reverse direction (region C). These modes in the reverse direction have different interference spacing but the same effective indices as in region A, as indicated in Table I, providing an additional consistency check.

Next we analyze the propagating modes, later returning to the unique properties of the modes in region $\mathrm{B}$, nonpropagating features, and diffraction fringes.

\section{B. Photonic waveguide modes}

The Fourier spectra in Fig. 6 exhibit broadened resonances due to the short decay length of the surface waves. In the Fourier transforms, attenuation produces a Lorentzian peak shape whose half width at half maximum (HWHM) is the absorption coefficient $\alpha$. In both TM and TE region A modes, $\mathrm{HWHM} \approx 0.05 k=7700 \mathrm{~cm}^{-1}$. These values are in very good agreement with transmission measurement on ITO films. ${ }^{12,13}$ This is a confirmation that the observed waves actually propagate through the ITO layer.

We use the asymmetric slab waveguide model, as presented in Ref. 21, and insert the measured film thickness and optical parameters for ITO and glass, given in Sec. II. For these values the slab model predicts two guided modes for both TM and TE polarizations, and also provides theoretical predictions for the effective indices, which can now be compared with the experimental results. Such a comparison is presented in Fig. 7. We find that the theoretical effective indices are consistent with those obtained experimentally. The predictions also nicely show the observed index difference for the two thicknesses available in the structure.

\section{Mode amplitudes and phases}

Diffraction is the mechanism through which light is coupled into the ITO thin film. A model based on Fraunhofer (farfield) diffraction can be used to confirm this. Waves incident on the slit are diffracted through angle $\beta$ into the ITO layer, with in-plane wave number $k n_{\text {ITO }} \sin \beta$. The modes resonant in the waveguide structure are captured and have wave number $k N_{j} .{ }^{21}$ Thus, the diffraction angle of the $j$ th mode is related to the effective index by

$$
\sin \beta_{j}=N_{j} / n_{\mathrm{ITO}}
$$

The Fraunhofer (far-field) equation gives the amplitude distribution of a single slit illuminated with normal, coherent plane waves as

$$
E_{j}=E_{0} \operatorname{sinc}\left(k W N_{j} / 2 n_{\mathrm{ITO}}\right),
$$

where $\operatorname{sinc} x \equiv \sin x / x, W$ is the slit width, ${ }^{22}$ and the relation in Eq. (2) has been used to eliminate the angle $\beta$. From Fig. 3(a), the slit is $375 \mathrm{~nm}$ wide for region A and $390 \mathrm{~nm}$ for region $B$.

The ratio of two mode amplitudes, $E_{1} / E_{2}$, can be measured from the Fourier transform, Fig. 6, using the relationships between intensity and amplitude from the interference model, given in Table I. The ratio can also be calculated from the effective indices, independently measured from the Fourier transform. Comparing these two methods, the measured amplitude ratio and effective-index calculated ratio are found to be in good agreement. In addition, diffraction can explain why the primary propagating wave interference maxima are not coincident with the slit edge. Following an argument similar to Ref. 23, light diffracted into the ITO layer at angle $\beta_{j}$ is reflected at the ITO-glass boundary. That ray returns to the ITO-vacuum surface a distance $\Delta y_{j}=2 t[1-$ $\left.\left(N_{j} / n_{\mathrm{ITO}}\right)^{2}\right]^{-1 / 2}$ from the slit, eliminating the angle $\beta_{j}$ with Eq. (2). $\Delta y_{j}$ is the distance between the slit edge and the primary maximum. Using the effective indices measured from the Fourier spectrum, the computed distances are 0.6-1 $\mu \mathrm{m}$ in the dominant waveguide modes, consistent with the observed phase offsets in regions A and B of Figs. 3(b) and $3(\mathrm{c})$.

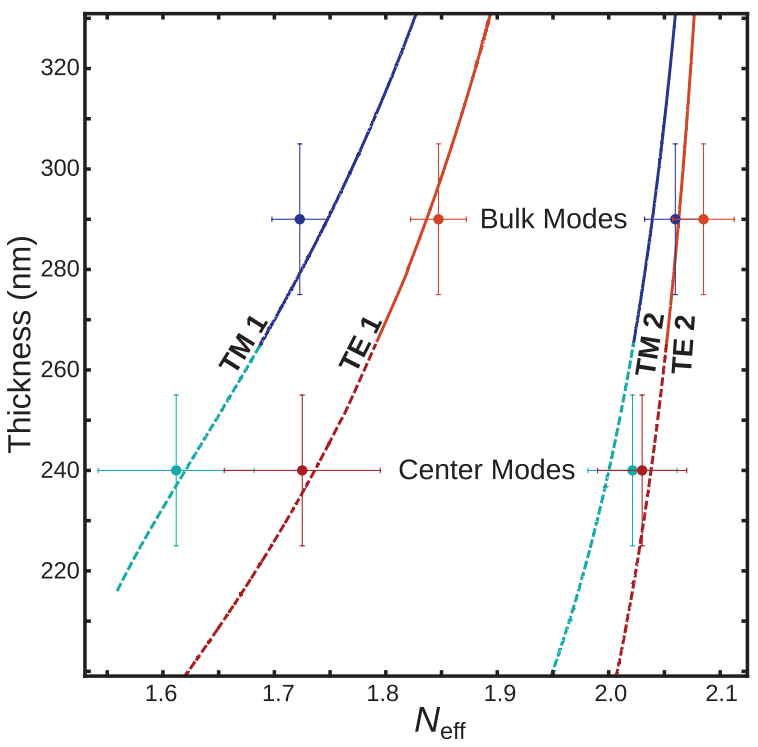

FIG. 7. (Color online) Allowed modes of the vacuum/ITO/glass waveguide model for ITO films of 200-320 nm. Experimental results determined from FFT indicated with error bars. 


\section{Region B modes}

The center region modes have some obvious differences from the surrounding region modes. The most striking observation is that the spacing of the interference pattern in region $\mathrm{B}$ is smaller than in region $\mathrm{A}$, indicating a smaller effective refractive index. This is explained by the fact that the guided wave also probes the space outside the geometrical volume of the ITO layer in the trench. As region B is thinner than region $\mathrm{A}$, the vacuum refractive index has a larger contribution to the effective index for the guided modes in region B. As a consequence, a lower effective index, a longer wavelength, and a larger spacing in the interference pattern result. This is in complete agreement with the asymmetric slab waveguide model, as compared in Fig. 7.

Another difference is the extremely rapid decay of the center modes, which have attenuation coefficients in the range $12-21 \times 10^{3} \mathrm{~cm}^{-1}$, two to three times larger than in region A. This is most likely due to lateral confinement of the trench region. Guided modes are best confined if the refractive index of the confining layer is greater than the surrounding materials. Since the effective indices of region B are less than in regions $\mathrm{A}$ and $\mathrm{C}$, the confinement in the center trench is "leaky" which results in a stronger damping of the propagating modes. ${ }^{21}$

Region B has two additional interesting features which contribute to increased background brightness. At the far edge, reverse modes are coupled in and interfere with the forward propagating waves. The result is that the region is generally brighter than the surrounding bulk surface but without a strong contrast in the interference pattern, as seen in the profiles of Fig. 4. Second, the surface of the trench was FIB milled, unlike regions $\mathrm{A}$ and $\mathrm{C}$. FIB milling modifies surfaces, changing surface roughness and disrupting the surface homogeneity due to gallium impact. ${ }^{24}$ The difference in surface roughness between the center and bulk surface regions is also visible in the one photon image, Fig. 3(a). These changes are generally limited to a shallow surface depth, so the effects of FIB milling on wave guiding deep in the ITO layer should be minimal.

\section{E. Local near fields and diffraction}

Although not the focus of this paper, it is worth briefly discussing other features of the two-photon photoemission images. Most notable are localized emission "hot spots" scattered across the ITO surface in Figs. 3(b) and 3(c), many of which are present in both polarizations. Comparing to Fig. 3(a), it seems that most of these excitations are due to surface irregularities. These sites produce localized near-field optical resonances which enhance the electromagnetic field. Similarly, the top edges of the slit and center region resonate with the incident light, enhancing photoemission. These edges are significantly narrower than the interference pattern maxima, indicating tightly confined electromagnetic fields.

Diffraction fringe fields extend around hot spot sites in the multiphoton PEEM micrographs and are significantly weaker under TE polarization. The occurrence of these fringes has been discussed in some detail in Ref. 25. The width of the fringe maxima varies with particle size and is projected further in the forward direction than in the reverse. These features clearly distinguish the waveguide modes from diffraction phenomena.
The fringe fields can be explained using Fraunhofer (farfield) diffraction around an opaque aperturelike object, which predicts a minima immediately surrounding the particles and gives a first maximum spacing $\Delta y \approx 3.83 \lambda A / \pi \sin 30^{\circ}$ in the forward direction, where $A$ is the aspect ratio of height to diameter. ${ }^{25}$ For $A \leqslant 1$, this formula predicts a variable spacing $\Delta y \lesssim 1 \mu \mathrm{m}$, consistent with the forward fringes observed in Fig. 3.

\section{F. Error analysis}

It is of interest to determine the potential accuracy of PEEM in this optical application, particularly for the experimentally observed optical parameters such as the refractive index, the absorption coefficient, and relative phase shifts. The microscope utilized in these experiments had a spatial resolution of $5 \mathrm{~nm}$ in earlier studies. ${ }^{16}$ In the current work, the low electron emission rates result in long exposure times which in turn lead to a degradation of the spatial resolution, as voltage stability, sample motion, and external fields have an increasing influence on the image.

From the experimental data we find that the Fourier transform evaluation provides interference pattern spacings with an accuracy of $3 \%$, the effective indices of the guided modes with $5 \%$, and the absorption coefficient with $9 \%$. Assuming that the laser wavelength, the angle of incidence, and the film thickness could — at least in principle_-be determined with significantly better accuracy, we conclude that the optical parameters can be determined with high precision in areas as small as a few microns, since the Fourier transform is obtained over that area.

The determination of relative phase shifts can be obtained from a direct image analysis of Fig. 4 once the periods are determined from the Fourier analysis. The slit edge can be taken as the reference position for this comparison. A basic analysis using fitted wave forms indicates that the two images provide an accuracy of $24 \mathrm{~nm}$ for the relative phase shifts of the two polarization modes depicted. This corresponds to corresponds to $\lambda / 17$.

We find that these results for local averages of the optical constants and the dynamic phase shifts in diffractive incoupling nicely demonstrate the potential for PEEM imaging in planar optics applications.

\section{CONCLUSION}

Transparent photonic waveguides can quantitatively be studied in PEEM at excellent spatial resolution. Our method utilizes multiphoton excitation processes to bridge the energy gap between work function and visible light.

In the current study photonic propagation of blue light was observed by a nonlinear two-photon coupling in the material induced by the high intensities available in ultrashort laser pulses. The nonlinear photoemission process also increased the imaging contrast. Highly redundant results produced a unique, high precision characterization. It appears that photoemission microscopy with its excellent spatial resolution is suitable to quantify phase shifts locally and under a wide range of conditions, i.e., at altered polarizations, in different surroundings and over a range of material properties. 
Our finding complements earlier work that has demonstrated the visualization of plasmon propagation in thin metallic films using a very similar approach. ${ }^{17-19}$ As photonic wave propagation is better understood and is subject to lower internal damping, the experimental results in photonics are more readily understood and can be analyzed at a much more quantitative level. It can be expected that extending the interferometric approach from the plasmonic to the photonic regime will eventually allow the observation of interactions between surface plasmon and guided photons, particularly the excitation and radiative decay of surface plasmons. Including a spectroscopic analysis of the photoelectrons in such experiments will make this description even more definitive.

The high contrast inherent to multiphoton photoemission is particularly well suited to nanoscale devices as it allows high precision determination of mode wavelength, amplitude, and phase. There are numerous applications where these parameters are of importance, ${ }^{26}$ as, for example, in high-confinement waveguides,${ }^{27}$ high sensitivity biochemical sensing devices, ${ }^{28}$ quantum coherent devices, ${ }^{29}$ and ultrafast optical switches. ${ }^{30}$ While phase sensitivity is one aspect not fully explored in the present analysis, the high contrast available in nonlinear PEEM micrographs allows for phase measurements to within $1 / 17$ th of a cycle. This can provide new experimental support to the design of metamaterials, ${ }^{31}$ where relative electromagnetic phase shifts play an important role.

\section{ACKNOWLEDMENT}

This research was funded by the US Department of Energy Basic Science Office under Contract No. DE-FG0210ER46406. *fit@pdx.edu

${ }^{1}$ C. B. Harris, N.-H. Ge, R. L. Lingle, J. D. McNeill, and C. M. Wong, Annu. Rev. Phys. Chem. 48, 711 (1997).

${ }^{2}$ O. Schmidt, G. Fecher, Y. Hwu, and G. Schönhense, Surf. Sci. 482-485, Part 1, 687 (2001).

${ }^{3}$ R. C. Word, J. P. S. Fitzgerald, and R. Könenkamp, Surf. Sci. 607, 148 (2013).

${ }^{4}$ M. Merschdorf, W. Pfeiffer, A. Thon, S. Voll, and G. Gerber, Appl. Phys. A: Mater. Sci. Process. 71, 547 (2000).

${ }^{5}$ A. Gloskovskii, D. Valdaitsev, S. Nepijko, G. Schönhense, and

B. Rethfeld, Surf. Sci. 601, 4706 (2007).

${ }^{6}$ R. Könenkamp, R. C. Word, J. P. S. Fitzgerald, A. Nadarajah, and S. D. Saliba, Appl. Phys. Lett. 101, 141114 (2012).

${ }^{7}$ R. Weiher and R. Ley, J. Appl. Phys. 37, 299 (1966).

${ }^{8}$ R. Schlaf, H. Murata, and Z. Kafafi, J. Electron Spectrosc. Relat. Phenom. 120, 149 (2001).

${ }^{9}$ G. Naik, J. Kim, and A. Boltasseva, Opt. Mater. Express 1, 1090 (2011).

${ }^{10}$ S. Franzen, J. Phys. Chem. C 112, 6027 (2008).

${ }^{11}$ S. H. Brewer and S. Franzen, J. Phys. Chem. B 106, 12986 (2002).

${ }^{12}$ D. Davazoglou, Thin Solid Films 302, 204 (1997).

${ }^{13}$ V. Craciun, C. Chiritescua, F. Kelly, and R. Singh, J. Optoelectron. Adv. Mater. 4, 21 (2002).

${ }^{14}$ H. Kim, J. Horwitz, G. Kushto, A. Pique, Z. Kafafi, C. Gilmore, and D. Chrisey, J. Appl. Phys. 88, 6021 (2000).

${ }^{15}$ G. F. Rempfer, J. Appl. Phys. 67, 6027 (1990).

${ }^{16}$ R. Könenkamp, R. C. Word, G. F. Rempfer, T. Dixon, L. Almaraz, and T. Jones, Ultramicroscopy 110, 899 (2010).

${ }^{17}$ A. Kubo, N. Pontius, and H. Petek, Nano Lett. 7, 470 (2007).
${ }^{18}$ C. Lemke, T. Leißner, S. Jauernik, A. Klick, J. Fiutowski, J. Kjelstrup-Hansen, H. Rubahn, and M. Bauer, Opt. Express 20, 12877 (2012).

${ }^{19}$ L. Zhang, A. Kubo, L. Wang, H. Petek, and T. Seideman, Phys. Rev. B 84, 245442 (2011).

${ }^{20}$ J. Park, P. Ambwani, M. Manno, N. Lindquist, P. Nagpal, S. Oh, C. Leighton, and D. Norris, Adv. Mater. 24, 3988 (2012).

${ }^{21}$ A. Yariv, Optical Electronics (Saunders College Publishing, Philadelphia, 1991).

${ }^{22}$ F. Jenkins and H. White, Fundamentals of Optics (McGraw-Hill, New York, 2001).

${ }^{23}$ D. Rosenblatt, A. Sharon, and A. Friesem, IEEE J. Quantum. Electron. 33, 2038 (1997).

${ }^{24}$ J. McCaffrey, M. Phaneuf, and L. Madsen, Ultramicroscopy 87, 97 (2001).

${ }^{25}$ L. I. Chelaru, M. Horn-von Hoegen, D. Thien, and F. J. Meyer zu Heringdorf, Phys. Rev. B 73, 115416 (2006).

${ }^{26}$ O. Benson, Nature (London) 480, 193 (2011).

${ }^{27}$ R. Oulton, V. Sorger, D. Genov, D. Pile, and X. Zhang, Nat. Photon. 2, 496 (2008).

${ }^{28}$ F. De Angelis, M. Patrini, G. Das, I. Maksymov, M. Galli, L. Businaro, L. Andreani, and E. Di Fabrizio, Nano Lett. 8, 2321 (2008).

${ }^{29}$ A. Faraon, I. Fushman, D. Englund, N. Stoltz, P. Petroff, and J. Vuckovic, Opt. Express 16, 12154 (2008).

${ }^{30}$ T. Utikal, M. I. Stockman, A. P. Heberle, M. Lippitz, and H. Giessen, Phys. Rev. Lett. 104, 113903 (2010).

${ }^{31}$ A. Sihvola, Metamaterials 1, 2 (2007). 